\title{
Nonlinear inelastic electron scattering revealed by plasmon-enhanced electron energy-loss spectroscopy
}

\author{
Chun Kai Xü ${ }^{1,2}$, Wen Jie Liư ${ }^{1}$ Pan Ke Zhang ${ }^{1,2}$, Meng $\mathrm{Li}^{1,2}$, Han Jun Zhang ${ }^{1}$, Ke Zun Xu1, Yi Luo ${ }^{2,3 \star}$ \\ and Xiang Jun Chen ${ }^{1,2 \star}$
}

Electron energy-loss spectroscopy is a powerful tool for identifying the chemical composition of materials ${ }^{1-5}$. It relies mostly on the measurement of inelastic electrons, which carry specific atomic or molecular information. Inelastic electron scattering, however, has a very low intensity, often orders of magnitude weaker than that of elastically scattered electrons. Here, we report the observation of enhanced inelastic electron scattering from silver nanostructures, the intensity of which can reach up to $60 \%$ of its elastic counterpart. A home-made scanning probe electron energy-loss spectrometer 6 was used to produce highly localized plasmonic excitations, significantly enhancing the strength of the local electric field of silver nanostructures. The intensity of inelastic electron scattering was found to increase nonlinearly with respect to the electric field generated by the tip-sample bias, providing direct evidence of nonlinear electron scattering processes.

Figure 1a gives a schematic drawing of the scanning probe electron energy-loss spectroscopy (SP-EELS) technique used in this study, the details of which can be found elsewhere ${ }^{6}$. Briefly, it consists of a tip-sample system and a toroidal electron energy analyser (TEEA). The experimental arrangement is sketched in Fig. 1b. A tip made from a $0.42 \mathrm{~mm}$ tungsten wire by electrochemical etching is approached to a distance of micrometres from the grounded sample surface, which is prepared by evaporating a $30 \mathrm{~nm}$ thin film of Ag on freshly cleaved highly ordered pyrolytic graphene (HOPG). Silver structures with dimensions of tens of nanometres are observed on the sample surface, as illustrated in the inset of Fig. 1b. Electrons are field-emitted from the tip when a negative tip voltage $V_{\mathrm{t}}$ of hundreds of volts is applied, and surface plasmon resonance (SPR) of the Ag nanostructures is excited by the field-emission electrons under a strong electric field introduced by the tip-sample bias. The backscattered electrons from the sample surface are collected and analysed by the TEEA. In this way the electron energy-loss spectroscopy (EELS) under a certain tip-sample distance and tip voltage can be acquired.

It should be mentioned that EELS has been applied for decades to detect surface plasmons ${ }^{1,7-14}$, and in combination with a scanning transmission electron microscope (STEM) it is capable of mapping the spatial variation of SPRs at the nanoscale $e^{11-14}$. The energy-loss feature of Ag systems has been studied extensively by EELS, including low-energy backscattering EELS (refs 1,10) and high-energy transmission EELS (refs 11,13,14). A typical EELS spectrum of $\mathrm{Ag}$ nanostructures is shown in Fig. 1c, which was

obtained at a tip-sample distance of $114 \mu \mathrm{m}$ with a tip voltage of $-246 \mathrm{~V}$ and a sample current of $10 \mathrm{pA}$. The energy-loss peak located at about $3.7 \mathrm{eV}$ is associated with the SPR excitation of Ag. Our spectrum is in excellent agreement with those reported by Palmer's group, who also used scanning probe electron spectroscopy to investigate the EELS of Ag surfaces ${ }^{15,16}$. In their experiments, a tip withdrawn a distance from the tunnelling region was invoked as a field-emission electron source when a bias voltage of hundreds of volts was applied. The energy of backscattered electrons was analysed by means of a hemispherical deflector. One can see a common feature from these spectra-namely, the Ag SPR excitation peak (inelastic) is much weaker than the main elastic scattering (ES) peak. This accords well with the general pattern of the electron scattering processes.

We have made measurements at three different tip-sample distances: $92 \mu \mathrm{m}, 114 \mu \mathrm{m}$ and $150 \mu \mathrm{m}$, respectively. At each distance, the tip voltage is increased step by step, and the EELS spectra at different electric fields are obtained. Figure 2a shows five EELS spectra acquired at a tip-sample distance of $150 \mu \mathrm{m}$ with different tip voltages. It can be seen that increasing the tip bias has a significant impact on the intensity of the SPR peak, which can be enhanced drastically when the value of $V_{\mathrm{t}}$ goes beyond $320 \mathrm{~V}$. It is quite striking to observe that the intensity of the SPR peak can even reach a value as high as $60 \%$ of the ES peak. The full data acquired at three different tip-sample distances are presented in Fig. 2b, illustrating the intensity as a function of the energy loss and tip voltage. One can see that for all three tip-sample distances the intensity of the SPR peak clearly increases with the tip voltage.

According to surface electronic excitation theory ${ }^{1}$, the SPRs are mainly excited by the long-range dipole scattering of incident electrons with momentum transfer parallel to the sample surface. Therefore, in the backscattering condition the SPR excitation must be a multiple-scattering process of large-angle elastic scattering followed by near-zero-angle dipole scattering (Fig. 3). In this case, the count rate of inelastic electrons with the energy loss of SPR excitation $\left(n_{\mathrm{SPR}}\right)$ can be expressed as follows:

$$
n_{\mathrm{SPR}} \propto W_{\mathrm{SPR}} W_{\mathrm{els}} I_{0}
$$

where $W_{\text {els }}$ and $W_{\text {SPR }}$ are the transition probabilities of elastic scattering and dipole scattering, respectively, and $I_{0}$ is the intensity of the incident electron beam. As the count rate of the elastic scattering

${ }^{1}$ Department of Modern Physics, University of Science and Technology of China, Hefei 230026, China, ${ }^{2}$ Hefei National Lab for Physical Science at Microscale and Synergetic Innovation Center of Quantum Information Quantum Physics, University of Science and Technology of China, Hefei 230026, China, ${ }^{3}$ Department of Theoretical Chemistry and Biology, Royal Institute of Technology, S-106 91 Stockholm, Sweden.

*e-mail: yiluo@ustc.edu.cn; xjun@ustc.edu.cn 


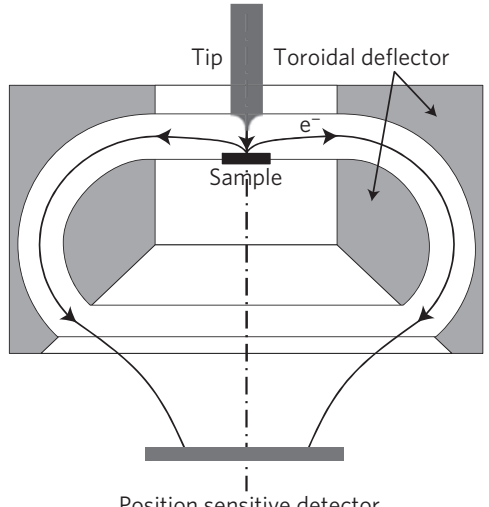

Position sensitive detector

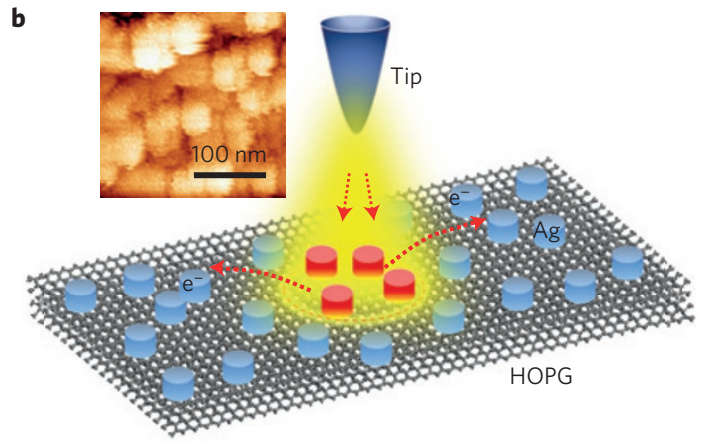

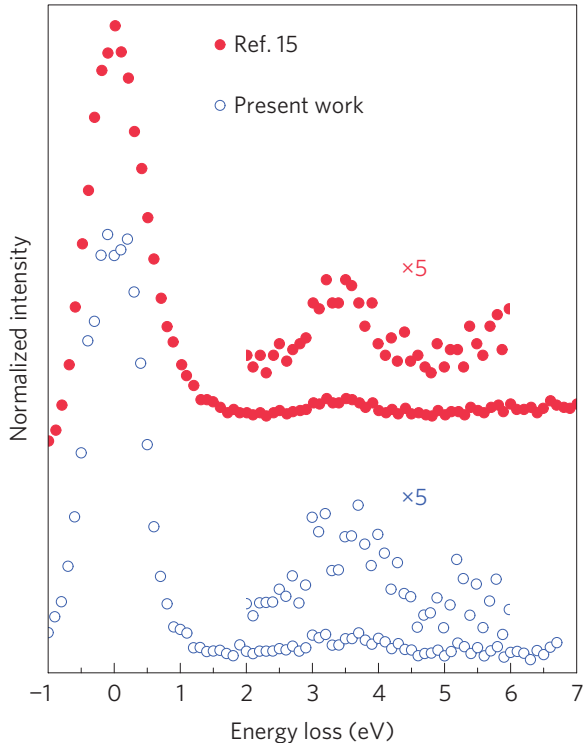

Figure 1 | Experimental arrangement. a, Schematic drawing of the scanning probe electron energy-loss spectroscopy (SP-EELS) technique, which consists of a tip-sample system and a toroidal electron energy analyser (TEEA). b. Experimental arrangement. A tip is approached to a distance of micrometres from a grounded highly ordered pyrolytic graphene (HOPG) surface carrying Ag nanostructures (illustrated in the inset). Electrons are field-emitted from the tip when a negative tip voltage $V_{t}$ of hundreds of volts is applied, and the surface plasmon resonance (SPR) of the Ag nanostructures is excited by the field-emitted electrons under a strong electric field introduced by the tip-sample bias. The backscattered electrons are collected and analysed by the TEEA. c, EELS spectra acquired at a tip-sample distance of $114 \mu \mathrm{m}$ with a tip voltage of $-246 \mathrm{~V}$ and sample current of $10 \mathrm{pA}$ (open blue circles) for a $30 \mathrm{~nm}$ thick $\mathrm{Ag}$ film on HOPG. An energy-loss peak located at approximately $3.7 \mathrm{eV}$ is clearly observed, which is associated with the SPR excitation of Ag. The spectrum is compared with that obtained at a tip voltage of $-170 \mathrm{~V}$ and a sample current of $10 \mathrm{nA}$ for a $200 \mathrm{~nm}$ thick Ag film on HOPG by Palmer et al. (solid red circles) ${ }^{15}$.

a

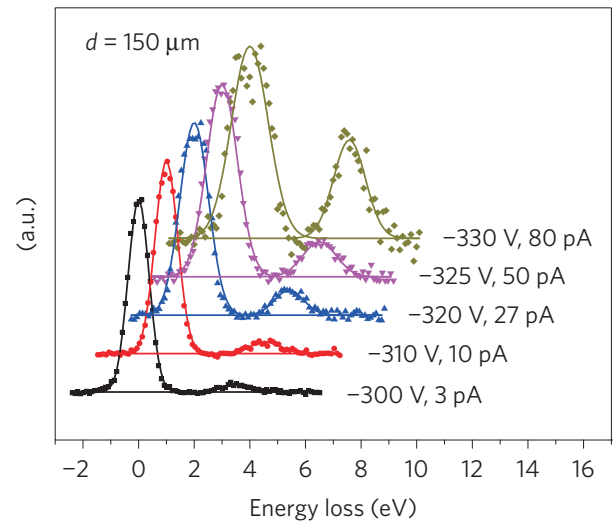

b

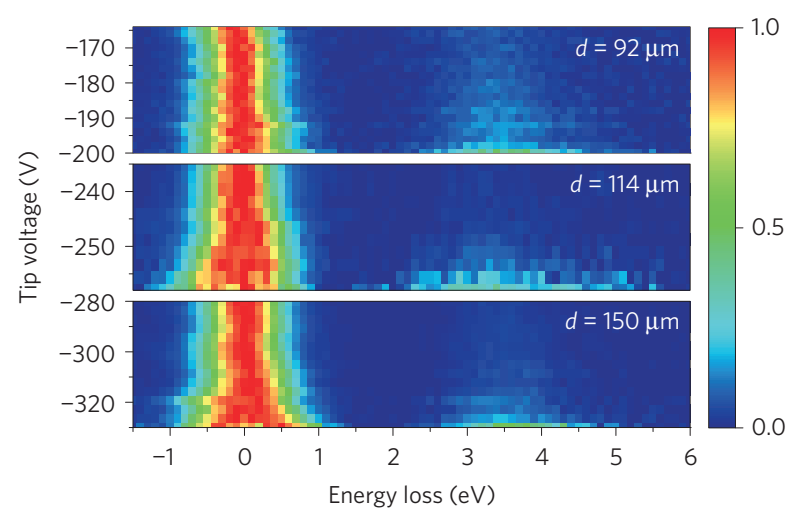

Figure 2 | Experimental data. a, Five EELS spectra acquired at a tip-sample distance of $150 \mu \mathrm{m}$ with different tip voltages and sample currents. Gaussian function fitted curves of the elastic scattering peaks and surface plasmon resonance peaks are also shown as solid lines. $\mathbf{b}$, Experimental data for the $\mathrm{Ag}$ nanostructures at three different tip-sample distances shown as tip voltage plotted against energy loss. For comparison, all the EELS spectra obtained have been background subtracted using a polynomial function and divided by the maximum of the elastic scattering peak.

electrons $\left(n_{\mathrm{els}}\right)$ is proportional to the product of $W_{\mathrm{els}}$ and $I_{0}$-namely, $n_{\mathrm{els}} \propto W_{\mathrm{els}} I_{0}$ - then $W_{\text {SPR }}$ can be expressed as

$$
W_{\mathrm{SPR}} \propto \frac{n_{\mathrm{SPR}}}{n_{\mathrm{els}}}=\mathrm{RI}
$$

where the relative intensity RI is defined as the area ratio of the SPR energy-loss peak to the ES peak in EELS. In the linear response regime, RI is independent both of the current $I_{0}$ and the strength of the electric field, as often observed in the experimental results reported so far.

In Fig. $4 a-c$, the RI values are plotted as a function of tip voltage at tip-sample distances of $92 \mu \mathrm{m}, 114 \mu \mathrm{m}$ and $150 \mu \mathrm{m}$, respectively. It can be found that, for each distance, when the tip voltage is low, RI, as expected, remains almost unchanged. 


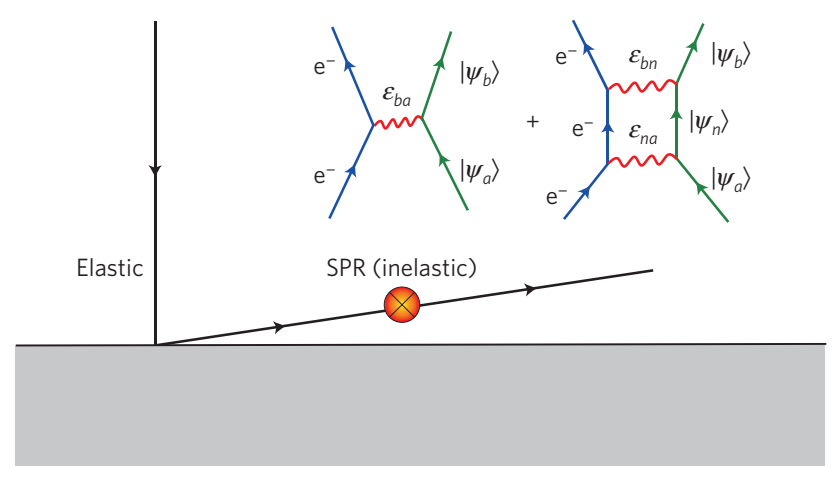

Figure 3 | The multiple-scattering process involved in SPR excitation. As the SPR is mainly excited by dipole scattering with the momentum transfer parallel to the surface, SPR excitation in the backscattering condition must be a multiple-scattering process consisting of a large-angle elastic scattering followed by a near-zero-angle dipole scattering. The inset shows the first- and second-order interactions involved in the dipole scattering.

However, when the tip voltage increases beyond a certain value, RI increases rapidly, indicating a strong enhancement of SPR excitation, and the dependence of RI on the tip voltage fits a quadratic curve fairly well (shown as a solid line). It is worth noting that at a larger tip-sample distance the increase of RI begins at a larger tip voltage. This suggests that the enhancement of SPR excitation should be attributed to the electric field rather than the tip voltage or the incident electron energy. According to the previous experimental observations of Palmer's group ${ }^{16}$ in the energy range of our experiments, the increase of the incident electron energy should actually cause a decrease of the SPR peak intensity, rather than the huge enhancement as observed here. The observation of the nonlinear electron scattering is further illustrated by the dependence of RI on the sample current at three tip-sample distances, which is shown in Fig. $4 \mathrm{~d}-\mathrm{f}$, respectively. In all these three figures, the RI values are no longer a constant, but increase nonlinearly with the sample currents. As the field emitted current is directly associated with the strength of the tip electric field, which is proportional to the field on the sample surface, these results further confirm the decisive role played by the electric field.

It could be argued that the angular range collected by the spectrometer might affect the spectral profile under different tip voltages. In our experiments, the energy loss of approximately $3.7 \mathrm{eV}$ is much smaller than the incident electron energy (tip voltage), therefore the change of the tip voltage should have very small influence on the angular collection range of the SPR energy-loss electron. This has been confirmed by simulating the trajectories of the backscattered inelastic electrons under our experimental conditions using charged particle optics (CPO) software (see Supplementary Methods for details).

Recently, the nonlinear processes induced by SPR have been revealed in different new spectroscopic studies. For instance, it was observed in photo-induced near-field electron microscopy (PINEM), invented by Zewail and co-workers ${ }^{17-19}$, that the intensities of both electron energy-loss and energy-gain peaks became compatible with the zero-loss (elastic) peak under excitation of SPRs induced by ultrafast lasers. However, it should be emphasized that in their experiments the generation of SPRs and electron scattering come from two separate sources and the electron energy-loss spectra could still be treated by the linear response ${ }^{17,19}$. In contrast, both events in our set-up are caused only by a single electron process. In our experiments, the maximal incident current is $1 \mathrm{nA}$ and the area of the current spot on the sample surface is at least several hundred square micrometres. One can easily estimate that the average time interval for two subsequent incident electrons interacting with one Ag nanostructure (less than 0.01 square micrometre according to the inset in Fig. 1b) is more than $1 \mu \mathrm{s}$. Considering the fact that the typical damping time of the SPR is less than $1 \mathrm{ps}$, the possible involvement of two-electron processes can thus safely be ruled out. The observed huge enhancement of inelastic electron peaks can thus be attributed to the plasmon-assisted nonlinear electron scattering process.

As the value of RI directly reflects the process of the near-zeroangle dipole scattering, it can be described well by perturbation theory under the dipole approximation. Taking into account the
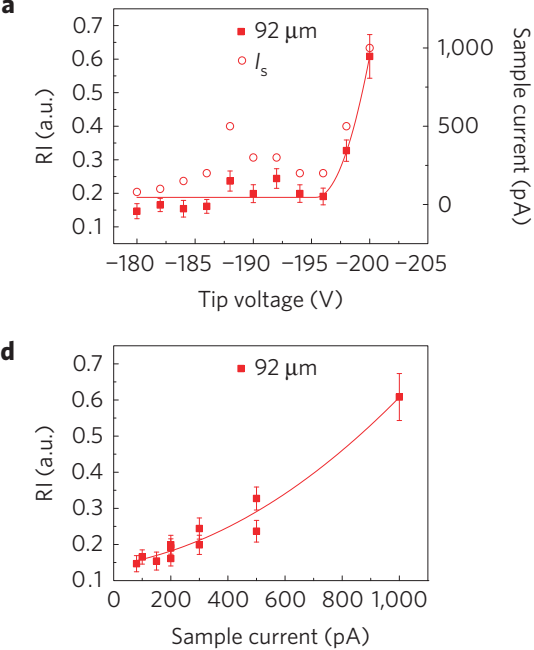

b

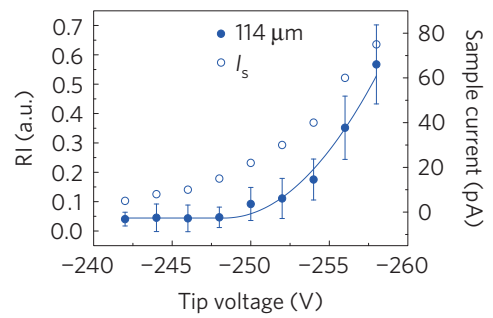

e

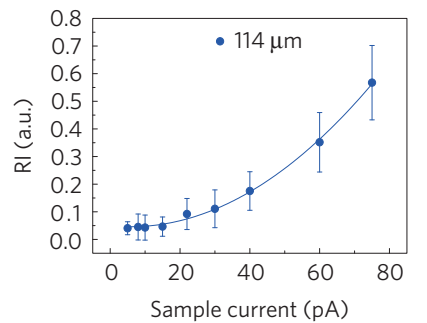

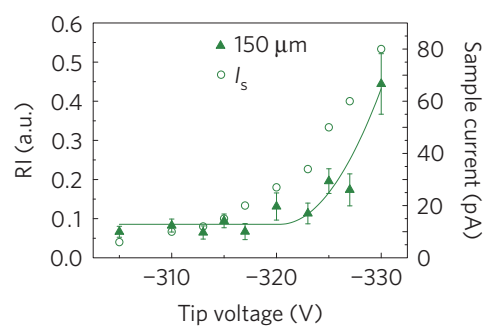

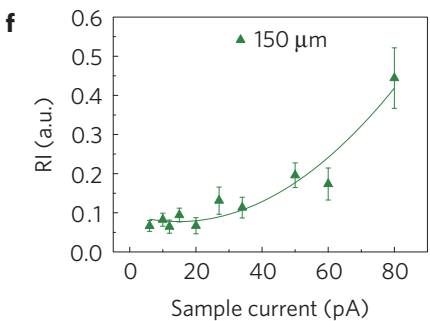

Figure 4 | Relative intensity of the SPR energy-loss peak for three tip-sample distances. a-c, Data with error bars shows relative intensity (RI, in arbitrary units (a.u.)) as a function of tip voltage at tip-sample distances of $92 \mu \mathrm{m}, 114 \mu \mathrm{m}$ and $150 \mu \mathrm{m}$ respectively; the sample current is also plotted as open circles for each distance. A marked increase of Rl is observed after the tip voltage increases beyond a certain value, indicating a strong enhancement associated with SPR excitation. A solid line with a constant value is fitted to the data before the sudden increase of RI, and a quadratic curve is employed afterwards. d-f, RI versus sample current. The solid lines are guides to the eyes. The error bars are the standard errors corresponding to peak deconvolution and background subtraction. 


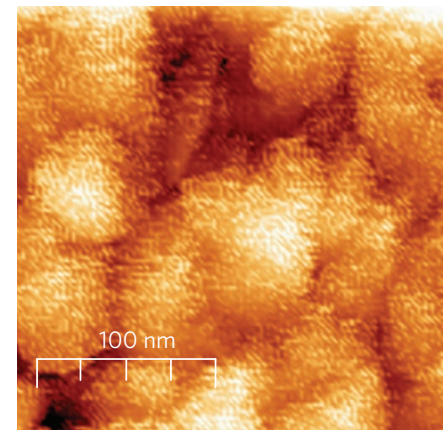

d

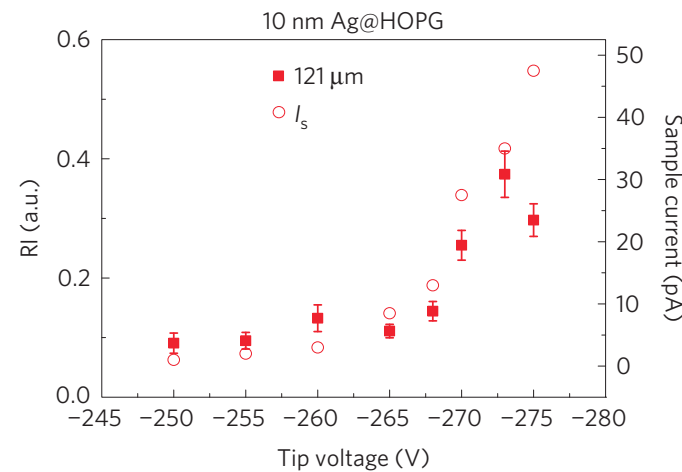

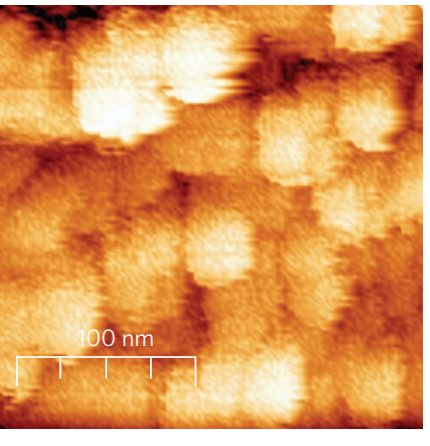

e

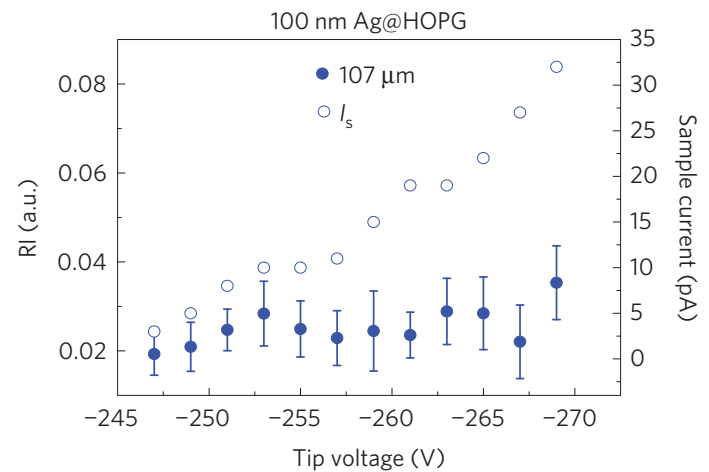

Figure 5 | Results of experiments on $10 \mathrm{~nm}$ and $100 \mathrm{~nm}$ thick Ag on HOPG. a-c, Topographies of sample surfaces of $10 \mathrm{~nm}, 30 \mathrm{~nm}$ and $100 \mathrm{~nm}$ thick Ag on HOPG, respectively. d,e, Dependences of relative intensity (RI, in arbitrary units (a.u.)) on tip voltage for $10 \mathrm{~nm}$ and $100 \mathrm{~nm} \mathrm{Ag@HOPG,} \mathrm{respectively,} \mathrm{plotted}$ as solid symbols with error bars. The sample currents are also plotted as open circles. A nonlinear dependence of RI on tip voltage is clearly observed for the $10 \mathrm{~nm}$ Ag@HOPG sample at a tip-sample distance of $121 \mu \mathrm{m}$, whereas no evident enhancement of RI can be observed for the 100 nm Ag@HOPG sample at a tip-sample distance of $107 \mu \mathrm{m}$. The error bars are the standard errors corresponding to peak deconvolution and background subtraction.

second-order interaction, as shown in the inset of Fig. 3, the transition probability can be expressed as follows

$$
\begin{aligned}
W_{\mathrm{ba}}= & W_{\mathrm{ba}}^{(1)}+W_{\mathrm{ba}}^{(2)} \propto\left|\left\langle b\left|\mathbf{E}_{1} \cdot \mathbf{D}_{\mathrm{ba}}\right| a\right\rangle\right|^{2} \\
& +\left|\sum_{n} \frac{\left\langle b\left|\mathbf{E}_{2} \cdot \mathbf{D}_{\mathrm{bn}}\right| n\right\rangle\left\langle n\left|\mathbf{E}_{1} \cdot \mathbf{D}_{\mathrm{na}}\right| b\right\rangle}{\Gamma_{n} / 2}\right|^{2}
\end{aligned}
$$

where $|a\rangle$ and $|b\rangle$ are the ground state and the final SPR state, $|n\rangle$ is an intermediate state with energy width $\Gamma_{n}, \mathbf{D}_{\mathrm{ba}}, \mathbf{D}_{\mathrm{bn}}$ and $\mathbf{D}_{\mathrm{na}}$ are the matrix elements of the dipolar moment operator for electron scattering, $\mathbf{E}_{1}$ and $\mathbf{E}_{2}$ are the electric field experienced by $|a\rangle$ and $|n\rangle$, and the sum over $n$ is over all possible intermediate states. In a normal situation, the second-order term $W_{\mathrm{ba}}^{(2)}$ is very weak and may be neglected. Therefore the transition probability is dominated by the first-order term $W_{\mathrm{ba}}^{(1)}$ :

$$
W_{\mathrm{ba}} \approx W_{\mathrm{ba}}^{(1)} \propto\left|\left\langle b\left|\mathbf{E}_{1} \cdot \mathbf{D}_{\mathrm{ba}}\right| a\right\rangle\right|^{2} \propto\left(\mu_{\mathrm{ba}} E_{1}\right)^{2}
$$

where $\mu_{\mathrm{ba}}$ is the transition dipole moment between the initial and final states. When an external electric field $\mathbf{E}_{\mathrm{ex}}$ is applied, $\mathbf{E}_{1}$ is a combination of $\mathbf{E}_{\mathrm{ex}}$ and the electric field $\mathbf{E}_{\omega}$ introduced by the incident electron. Under normal conditions, we have $E_{\text {ex }} \ll E_{\omega}$, so that $W_{\mathrm{ba}}$ often shows no dependence on the external field. However, in the present work, the probability of SPR excitation is enhanced enormously, indicating that the second-order term must be taken into account. According to the expression

$$
W_{\mathrm{ba}}^{(2)} \propto\left|\sum_{n} \frac{\left\langle b\left|\mathbf{E}_{2} \cdot \mathbf{D}_{\mathrm{bn}}\right| n\right\rangle\left\langle n\left|\mathbf{E}_{1} \cdot \mathbf{D}_{\mathrm{na}}\right| a\right\rangle}{\Gamma_{n} / 2}\right|^{2}
$$

all intermediate states $|n\rangle$ can in principle contribute to $W_{\mathrm{ba}}^{(2)}$. Obviously, the high density of intermediate states certainly helps to increase the value of $W_{\mathrm{ba}}^{(2)}$, which is actually what $\mathrm{Ag}$ nanostructures can provide. In particular, the exceptionally large transition and permanent dipolar moments make the final SPR state $|b\rangle$ much more important than others. As a good approximation, we can assume that $W_{\mathrm{ba}}^{(2)}$ is mostly contributed by one term

$$
W_{\mathrm{ba}}^{(2)} \propto\left|\frac{\left\langle b\left|\mathbf{E}_{2} \cdot \mathbf{D}_{b}\right| b\right\rangle\left\langle b\left|\mathbf{E}_{1} \cdot \mathbf{D}_{\mathrm{ba}}\right| a\right\rangle}{\Gamma_{b} / 2}\right|^{2} \propto\left(\frac{2 \mu_{b} E_{2}}{\Gamma_{b}}\right)^{2} W_{\mathrm{ba}}^{(1)}
$$

thus, the transition probability can be expressed as

$$
W_{\mathrm{ba}}=(1+\beta) W_{\mathrm{ba}}^{(1)}
$$

where $\beta \propto\left(\left(2 \mu_{b} E_{2}\right) / \Gamma_{b}\right)^{2}$ is the enhancement factor, $\mu_{b}$ is the permanent dipole moment of state $|b\rangle$, and $E_{2}$ is the electric field experienced by state $|b\rangle$. For the excited SPR state of the Ag nanostructure, the dipole moment is expected to be very large, and the local electric field $\left(E_{2}\right)$ is enhanced enormously by the SPR oscillation. The combination of these two factors will result in a huge enhancement factor $\beta$, similar to the cases of surface-enhanced Raman spectroscopy (SERS; refs 20,21) and tip-enhanced Raman spectroscopy (TERS; refs 22-25). In other words, the SPR peak of EELS should thus be significantly enhanced, and its transition probability should be quadratically dependent on the external field, as clearly confirmed by our experimental observations.

It is noted that with a similar experimental set-up, Palmer's group observed no enhancement of SPR excitation on the Ag surface ${ }^{16}$, although the strength of the electric field introduced by the tip bias in their experiments was much larger than ours. This might be due to the difference in the samples employed. The samples used in their experiments were prepared by evaporating $200 \mathrm{~nm}$ thick Ag on HOPG, which could result in a relatively flat Ag surface, whereas small islands of $\mathrm{Ag}$ nanostructures are clearly formed in our 
samples. To verify this, we prepared two different Ag samples with different thicknesses, $10 \mathrm{~nm}$ and $100 \mathrm{~nm}$, on HOPG respectively, and repeated the same experiments. The topographies of the samples and the dependences of RI on the tip voltage are shown in Fig. 5. For the 10 nm Ag@HOPG, we again observed nonlinear enhancement of RI when the tip voltage goes beyond $-265 \mathrm{~V}$ (Fig. 5d). However, similar to the results of Palmer's group ${ }^{16}$, an increase of RI has not been observed for $100 \mathrm{~nm}$ Ag@HOPG (Fig. 5e). One most noticeable difference between samples of $10 \mathrm{~nm}$ (as well as $30 \mathrm{~nm}$ ) and $100 \mathrm{~nm}$ thick $\mathrm{Ag}$ is that the former have a much rougher surface and can form more gaps among nanoparticles, as illustrated in Fig. $5 \mathrm{a}-\mathrm{c}$. In other words, the thinner Ag samples (10 $\mathrm{nm}$ and $30 \mathrm{~nm}$ ) employed here contain more 'hot spots', which are known to favour the generation of the strong plasmon fields, as often demonstrated in the SERS measurements ${ }^{20,21}$. The lack of a sufficient number of 'hot spots' in the thick Ag film (100 nm) thus makes it incapable of producing nonlinear electron scattering.

The observation of nonlinear electron scattering lays the foundation for a new spectroscopic technology, namely nonlinear electron scattering spectroscopy (NESS). It is certainly useful for studying the dynamics of plasmon generation in metal surfaces. More importantly, it offers a powerful tool to study interfacial systems, such as atoms or molecules adsorbed on metal nanoparticles. The involvement of the nonlinear electron scattering process will significantly enhance the signal-to-noise ratio and improve the spectral resolution of the adsorbates. From the pure plasmon-based spectroscopy viewpoint, plasmons generated by electron excitation in an STM have some unique features. As demonstrated in a recent experimental and theoretical study on the electroluminescence of molecules in an STM (refs 26,27), a plasmon in a nanogap can behave like a highly confined ultra-fast laser source able to produce hot luminance, and even upconversion. The same concept can certainly be applied in our SP-EELS technique as a means to improve its spatial and spectral resolution.

\section{Methods}

Data analysis. For each measured EELS spectrum, background subtraction using a polynomial function ${ }^{16}$ was first performed. The spectrum was then normalized by the height of the ES peak for comparison. Here we used the maximum of the ES peak instead of the sample current to scale the data, because in our experiment, as the sample current increases to a large value, a saturation effect in the position-sensitive detector employed in the spectrometer would emerge, resulting in a decrease of the detection efficiency of the apparatus. Gaussian functions were used to fit the ES peak and SPR energy-loss peak respectively, and the relative intensity of the SPR peak was calculated from the ratio of these two fitted Gaussian peak areas.

Received 28 April 2013; accepted 30 June 2014; published online 10 August 2014

\section{References}

1. Rocca, M. Low-energy EELS investigation of surface electronic excitations on metals. Surf. Sci. Rep. 22, 1-71 (1995).

2. Suenaga, K. et al. Element-selective single atom imaging. Science 290, 2280-2282 (2000).

3. Varela, M. et al. Spectroscopic imaging of single atoms within a bulk solid. Phys. Rev. Lett. 92, 95502 (2004).

4. Kimoto, K. et al. Element-selective imaging of atomic columns in a crystal using STEM and EELS. Nature 450, 702-704 (2007).

5. Colliex, C. et al. Capturing the signature of single atoms with the tiny probe of a STEM. Ultramicroscopy 123, 80-89 (2012).

6. Xu, C. K. et al. Spatially resolved scanning probe electron energy spectroscopy for Ag islands on a graphite surface. Rev. Sci. Instrum. 80, 103705 (2009).
7. Lucas, A. A. \& Sunjić, M. Fast-electron spectroscopy of surface excitations. Phys. Rev. Lett. 26, 229-232 (1971).

8. Batson, P. E. Surface plasmon coupling in clusters of small spheres. Phys. Rev. Lett. 49, 936-940 (1982).

9. Ding, Z. J., Li, H. M., Pu, Q. R., Zhang, Z. M. \& Shimizu, R. Reflection electron energy loss spectrum of surface plasmon excitation of Ag: A Monte Carlo study. Phys. Rev. B 66, 085411 (2002).

10. Savio, L., Vattuone, L. \& Rocca, M. Surface plasmon dispersion on sputtered and nanostructured Ag (001). Phys. Rev. B 67, 045406 (2003).

11. Nelayah, J. et al. Mapping surface plasmons on a single metallic nanoparticle. Nature Phys. 3, 348-353 (2007).

12. Chu, M. W., Chen, C. H., García de Abajo, F. J., Deng, J. P. \& Mou, C. Y. Surface exciton polaritons in individual Au nanoparticles in the far-ultraviolet spectral regime. Phys. Rev. B 77, 245402 (2008).

13. Koh, A. L. et al. Electron energy-loss spectroscopy (EELS) of surface plasmons in single silver nanoparticles and dimers: Influence of beam damage and mapping of dark modes. ACS Nano 3, 3015-3022 (2009).

14. Nicoletti, O. et al. Three-dimensional imaging of localized surface plasmon resonances of metal nanoparticles. Nature 502, 80-84 (2013).

15. Palmer, R. E., Eves, B. J., Festy, F. \& Svensson, K. Scanning probe energy loss spectroscopy. Surf. Sci. 502-503, 224-231 (2002).

16. Pulisciano, A., Park, S. J. \& Palmer, R. E. Surface plasmon excitation of Au and Ag in scanning probe energy loss spectroscopy. Appl. Phys. Lett. 93, 213109 (2008).

17. Barwick, B., Flannigan, D. J. \& Zewail, A. H. Photon-induced near-field electron microscopy. Nature 462, 902-906 (2009).

18. Park, S. T., Lin, M. \& Zewail, A. H. Photon-induced near-field electron microscopy (PINEM): Theoretical and experimental. New J. Phys. 12, 123028 (2010)

19. Yurtsever, A., Veen, R. M. \& Zewail, A. H. Subparticle ultrafast spectrum imaging in 4d electron microscopy. Science 335, 59-64 (2012).

20. Camden, J. P. et al. Probing the structure of single-molecule surface-enhanced Raman scattering hot spots. J. Am. Chem. Soc. 130, 12616-12617 (2008).

21. Cang, H. et al. Probing the electromagnetic field of a 15-nanometre hotspot by single molecule imaging. Nature 469, 385-388 (2011).

22. Pettinger, B., Ren, B., Picardi, G., Schuster, R. \& Ertl, G. Nanoscale probing of adsorbed species by tip-enhanced Raman spectroscopy. Phys. Rev. Lett. 92, 096101 (2004)

23. Neacsu, C. C., Dreyer, J., Behr, N. \& Raschke, M. B. Scanning-probe Raman spectroscopy with single-molecule sensitivity. Phys. Rev. B 73, 193406 (2006)

24. Lal, S., Link, S. \& Halas, N. J. Nano-optics from sensing to waveguiding. Nature Photon. 1, 641-648 (2007).

25. Kawata, S., Inouye, Y. \& Verma, P. Plasmonics for near-field nano-imaging and superlensing. Nature Photon. 3, 388-394 (2009).

26. Dong, Z. C. et al. Generation of molecular hot electroluminescence by resonant nanocavity plasmons. Nature Photon. 4, 50-54 (2010).

27. Tian, G., Liu, J. C. \& Luo, Y. Density-matrix approach for the electroluminescence of molecules in a scanning tunneling microscope. Phys. Rev. Lett. 106, 177401 (2011)

\section{Acknowledgements}

This work was partly supported by the National Basic Research Program of China (Grant Nos. 2010CB923301 and 2010CB923304), the National Science Foundation of China (Grant Nos. 10404026 and 20925311) and the MOE 211 project.

\section{Author contributions}

W.J.L. and P.K.Z. contributed equally to this work. X.J.C. and K.Z.X. initiated the study. C.K.X., Y.L. and X.J.C. supervised the project. C.K.X. and X.J.C. designed the experiments. W.J.L., P.K.Z., M.L. and H.J.Z. performed the experiments. C.K.X., W.J.L., P.K.Z. and X.J.C. analysed the data. Y.L. proposed the theoretical model. C.K.X., Y.L. and X.J.C. interpreted the experiments and wrote the manuscript.

\section{Additional information}

Supplementary information is available in the online version of the paper. Reprints and permissions information is available online at www.nature.com/reprints.

Correspondence and requests for materials should be addressed to Y.L. or X.J.C.

\section{Competing financial interests}

The authors declare no competing financial interests. 